\title{
In Vitro Activity of Novel Antifungal Olorofim against Filamentous Fungi and Comparison to Eight Other Antifungal Agents
}

\author{
Ourania Georgacopoulos ${ }^{1, *(\mathbb{D})}$, Natalie S. Nunnally ${ }^{1}$, Eric M. Ransom ${ }^{2}$, Derek Law ${ }^{3}$, Mike Birch ${ }^{3}$, \\ Shawn R. Lockhart ${ }^{1}$ and Elizabeth L. Berkow ${ }^{1}$ \\ 1 Mycotic Diseases Branch, Centers for Disease Control and Prevention, Atlanta, GA 30333, USA; \\ nua5@cdc.gov (N.S.N.); gyi2@cdc.gov (S.R.L.); kuu4@cdc.gov (E.L.B.) \\ 2 Association of Public Health Laboratories, Silver Springs, MD 20910, USA; ericmransom@wustl.edu \\ $3 \quad$ F2G Ltd., Manchester M30 0LX, UK; dlaw@F2G.com (D.L.); mbirch@F2G.com (M.B.) \\ * Correspondence: orf1@cdc.gov
}

check for updates

Citation: Georgacopoulos, O.; Nunnally, N.S.; Ransom, E.M.; Law, D.; Birch, M.; Lockhart, S.R.; Berkow, E.L. In Vitro Activity of Novel Antifungal Olorofim against Filamentous Fungi and Comparison to Eight Other Antifungal Agents. J. Fungi 2021, 7, 378. https://doi.org/ 10.3390/jof7050378

Academic Editor: David S. Perlin

Received: 7 April 2021

Accepted: 8 May 2021

Published: 12 May 2021

Publisher's Note: MDPI stays neutral with regard to jurisdictional claims in published maps and institutional affiliations.

Copyright: (c) 2021 by the authors. Licensee MDPI, Basel, Switzerland. This article is an open access article distributed under the terms and conditions of the Creative Commons Attribution (CC BY) license (https:/ / creativecommons.org/licenses/by/ $4.0 /)$.
Abstract: Olorofim is a novel antifungal drug that belongs to the orotomide drug class which inhibits fungal dihydroorotate dehydrogenase (DHODH), thus halting pyrimidine biosynthesis and ultimately DNA synthesis, cell growth and division. It is being developed at a time when many invasive fungal infections exhibit antifungal resistance or have limited treatment options. The goal of this study was to evaluate the in vitro effectiveness of olorofim against a large collection of recently isolated, clinically relevant American mold isolates. In vitro antifungal activity was determined for 246 azole-susceptible Aspergillus fumigatus isolates, five A. fumigatus with $\mathrm{TR}_{34} / \mathrm{L} 98 \mathrm{H}$-mediated resistance, 19 Rhizopus species isolates, 21 Fusarium species isolates, and one isolate each of six other species of molds. Olorofim minimum inhibitory concentrations (MICs) were compared to antifungal susceptibility testing profiles for amphotericin B, anidulafungin, caspofungin, isavuconazole, itraconazole, micafungin, posaconazole, and voriconazole. Olorofim MICs were significantly lower than those of the echinocandin and azole drug classes and amphotericin B. A. fumigatus wild type and resistant isolates shared the same MIC50 $=0.008 \mu \mathrm{g} / \mathrm{mL}$. In non-Aspergillus susceptible isolates (MIC $\leq 2 \mu \mathrm{g} / \mathrm{mL}$ ), the geometric mean (GM) MIC to olorofim was $0.54 \mu \mathrm{g} / \mathrm{mL}$ with a range of $0.015-2 \mu \mathrm{g} / \mathrm{mL}$. Olorofim had no antifungal activity (MIC $\geq 2 \mu \mathrm{g} / \mathrm{mL}$ ) against $10 \%$ of the collection (31 in 297), including some isolates from Rhizopus spp. and Fusarium spp. Olorofim showed promising activity against $A$. fumigatus and other molds regardless of acquired azole resistance.

Keywords: olorofim; F901318; filamentous fungi; antifungal agents; Aspergillus fumigatus; Fusarium spp.; dark molds; multidrug resistance

\section{Introduction}

Invasive fungal infections (IFI) are increasing in prevalence globally in parallel with the increase in antifungal drug resistance, posing a serious challenge for healthcare providers [1-3]. Advances in immunosuppressive therapies have resulted in a growing population of immunocompromised patients who are vulnerable to fungal infections including individuals with HIV, cancer patients, organ transplant recipients, stem cell transplant recipients, and people with long-term hospitalizations [4]. Several pathogenic mold species have exhibited increasing antifungal resistance, chief among them Aspergillus fumigatus [5]. Invasive aspergillosis is the predominant invasive mold infection in patients, with Aspergillus increasingly resistant to first line triazole antifungals [6,7]. Other opportunistic molds, such as Rhizopus, Fusarium, and some dematiaceous mold species, also contribute to the burden of IFI in the healthcare setting and are increasingly refractory to available antifungal therapies [8].

Azoles, echinocandins, and the polyene amphotericin B are the only antifungals currently approved to treat invasive mold infections (IMIs). Although amphotericin B 
is often an effective therapy, it is associated with many adverse side effects including infusion-related reactions and nephrotoxicity [9]. Azole use can have side effects as well. Azoles are not $100 \%$ selective for binding to fungal targets (they can also bind to human CYP450) which can cause drug-drug interactions [10]. Long-term azole use can lead to acquired drug resistance, which is a concern as it leads to higher rates of treatment failure and longer hospital stays [11]. In addition to increasing azole resistance due to broader use of azoles in medicine, the use of azole compounds as agricultural fungicides has led to an increase in azole-resistant $A$. fumigatus, especially in Europe [11-15]. Challenges also exist in treating other rare or unusual mold infections. The paucity of new drug development in the antifungal pipeline has limited the treatment options for rare and difficult to treat molds such as the Mucorales, Fusarium and dematiaceous molds [16,17]. New drug development is needed to help combat these infections.

Olorofim (F2G Limited, Manchester, UK) is the first antifungal in a new drug class known as orotomides. Olorofim inhibits fungal dihydroorotate dehydrogenase (DHODH), halting pyrimidine biosynthesis and ultimately impacting DNA synthesis, cell growth and division. DHODH is a unique drug target rendering it less likely to be impacted by other acquired resistance mechanisms [18]. The fungal DHODH target differs significantly from human DHODH, minimizing target-based drug toxicity [19].

Olorofim displays antifungal activity against numerous species of molds including Penicillium spp., Coccidioides spp., Histoplasma capsulatum, Blastomyces dermatitidis, Fusarium spp., Scedosporium spp., Lomentospora prolificans, Scopulariopsis brevicaulis, and Aspergillus spp. [18-28]. The FDA has granted olorofim breakthrough therapy designation and orphan drug designation for treatment of invasive aspergillosis as well as for infections due to Lomentospora/Scedosporium, Scopulariopsis, and central nervous system (CNS) coccidioidomycosis. An open-label single-arm phase $2 \mathrm{~b}$ study for treatment of invasive fungal infections in patients who lack treatment options is underway (ClinicalTrials.gov Identifier: NCT03583164) [29].

In this study we assessed the in vitro efficacy of olorofim compared to MIC values of traditional antifungals using the Clinical and Laboratory Standards Institute (CLSI) reference method for antifungal susceptibility testing. Olorofim, azoles, echinocandins, and polyenes were tested against 297 contemporary mold isolates, including those which are refractory to currently available antifungal treatments.

\section{Materials and Methods}

A total of 297 mold isolates were tested, including azole-susceptible A. fumigatus ( $n=246)$, azole-resistant $A$. fumigatus with the $\mathrm{TR}_{34} / \mathrm{L} 98 \mathrm{H}(n=3)$ and TR34/L98H/S297T / F495I ( $n=2)$ mutations, Rhizopus microsporus $(n=3)$, Rhizopus oryzae $(n=16)$, Fusarium chlamydosporum $(n=1)$, Fusarium dimerum $(n=2)$, Fusarium moniliforme $(n=1)$, Fusarium oxysporum $(n=5)$, Fusarium verticillioides $(n=1)$, Fusarium solani species complex $(n=11)$, and rare and unusual mold species including Phialemonium curvatum $(n=1)$, Phaeoacremonium parasticum $(n=1)$, Sarocladium kiliense $(n=1)$, Ramularia species $(n=1)$, Metarrhizium anisopliae $(n=1)$, and Pleurostomophora richardsiae $(n=1)$. All mold isolates were received at CDC between 1998 and 2019 as part of ongoing surveillance and routine diagnostic testing and come from both clinical and environmental sources. All isolates were clinical except for $12 R$. microsporus and R. oryzae isolates which came from environmental sources, and 90\% of isolates were received between 2017 and 2019.

A. fumigatus isolates were screened for itraconazole, posaconazole, and voriconazole resistance using a plate assay as described [30]. Isolates with breakthrough growth on the plate were confirmed as resistant to azoles by standard broth microdilution according to the Clinical and Laboratory Standards Institute (CLSI) document M-38 [31]. Epidemiologic cutoff values for $A$. fumigatus of $\geq 2 \mu \mathrm{g} / \mathrm{mL}$ for itraconazole and $/ \mathrm{or} \geq 1 \mu \mathrm{g} / \mathrm{mL}$ for voriconazole were used to indicate reduced antifungal activity. Mutations in the Cyp51A gene were confirmed through gene sequencing as previously described [32]. 
Susceptibility testing of mold isolates against olorofim followed the methods described in CLSI reference standard M38-A2 (M38 Reference). F901318 (olorofim) powder was donated by F2G Limited (Manchester, UK). Dilutions of olorofim were prepared using DMSO and the concentration range set to $(0.0001-2 \mu \mathrm{g} / \mathrm{mL})$ in 2-fold serial dilutions. Olorofim drug plates were prepared using 96 -well polystyrene round-bottom microwell plates (Thermo Scientific, Item ID\# 262162) and $100 \mu \mathrm{L}$ of synthetic medium RPMI-1640 was added to the plates before dispensing the antifungal. The HP D300e Digital Dispenser, HP Dispensing Software and HP T8+ and HP D4+ Dispensing cassettes were used to dispense the predetermined dosages of olorofim [33]. Drug plates were prepared ahead of time and stored at $-80^{\circ} \mathrm{C}$ until day of use, when they were removed from the freezer and allowed to thaw in a $37^{\circ} \mathrm{C}$ incubator.

Isolates were cultured onto Sabouraud Dextrose (SabDex) agar slants and incubated for $2-7$ days at $35^{\circ} \mathrm{C}$. The suspension was prepared by adding $1 \mathrm{~mL}$ of Tween $20(2 \%$, prepared in molecular grade $\mathrm{H}_{2} \mathrm{O}$ ) to each agar slant. The solution was drawn off and heavier particles allowed to settle. Absorbance was determined by measuring the optical density at $530 \mathrm{~nm}$ (OD530) using a spectrophotometer and adjusted to the desired range with sterile water as follows: $\mathrm{OD}_{530}$ of 0.09 to 0.13 for Aspergillus and dematiaceous spp., and $\mathrm{OD}_{530}$ of $0.15-0.17$ for Fusarium and Rhizopus spp. For antifungal susceptibility testing the adjusted suspension was diluted 1:50 in RPMI-1640 broth and $0.1 \mathrm{~mL}$ of the diluted inoculum added to each well. A growth control well containing no antifungal agent was included, in addition to a well containing only the medium and nuclease free water as a negative control. Quality control isolates for each species tested are listed in Table 1.

Table 1. Quality control isolates used for each fungal organism tested.

\begin{tabular}{cc}
\hline Quality Control or Reference Strain & Species Tested \\
\hline Aspergillus fumigatus ATCC MYA-3626 & Fusarium spp., Phialemonium curvatum, Phaeoacremonium parasiticum, Sarocladium \\
Candida krusei ATCC 6258 & Ramularia sp., Metarrhizium anisopliae and Pleurostomophora richardsiae \\
Azole susceptible A. fumigatus B7698 & Rhizopus. \\
Azole resistant A. fumigatus B7815 & A.fumigatus \\
(CDC internal controls) & \\
\hline
\end{tabular}

Olorofim antifungal activity was compared with results for other drugs from antifungal susceptibility testing using custom frozen panels from TREK Diagnostics (Thermo Fisher Scientific, Oakwood Village, OH, USA), which included anidulafungin, caspofungin, isavuconazole, itraconazole, micafungin, posaconazole, and voriconazole. Amphotericin B susceptibility was determined using Etest (bioMérieux, France). MICs were read for amphotericin B and the azoles, whereas the MEC was read for the echinocandins as specified in the CLSI's M38 [31]. Endpoints were recorded at $24 \mathrm{~h}$ for Rhizopus spp. and $48 \mathrm{~h}$ for $A$. fumigatus, Fusarium spp., Phialemonium curvatum, Phaeoacremonium parasticum, Sarocladium kiliense, Ramularia species, Metarrhizium anisopliae, and Pleurostomophora richardsiae.

\section{Results}

3.1. CLSI Reference Method MIC Results for Olorofim, Azoles, Echinocandins and Amphotericin B against Molds

\subsubsection{Olorofim}

Olorofim MIC results for each species tested are listed in Table 2. Olorofim showed consistent antifungal activity when tested against azole-susceptible $A$. fumigatus isolates $\left(\mathrm{MIC}_{50}=0.008 \mu \mathrm{g} / \mathrm{mL}\right)$. All A. fumigatus isolates fell within a one to two dilution range of the $\mathrm{MIC}_{50}(0.008 \mu \mathrm{g} / \mathrm{mL})$ (Figure 1). The five azole-resistant $A$. fumigatus isolates with Cyp51A-associated point mutations had MIC values of $0.008 \mu \mathrm{g} / \mathrm{mL}$, the same as the $\mathrm{MIC}_{50}$ of azole-susceptible A. fumigatus isolates. 


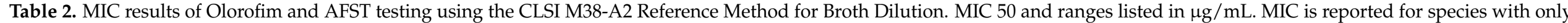
1 isolate tested.

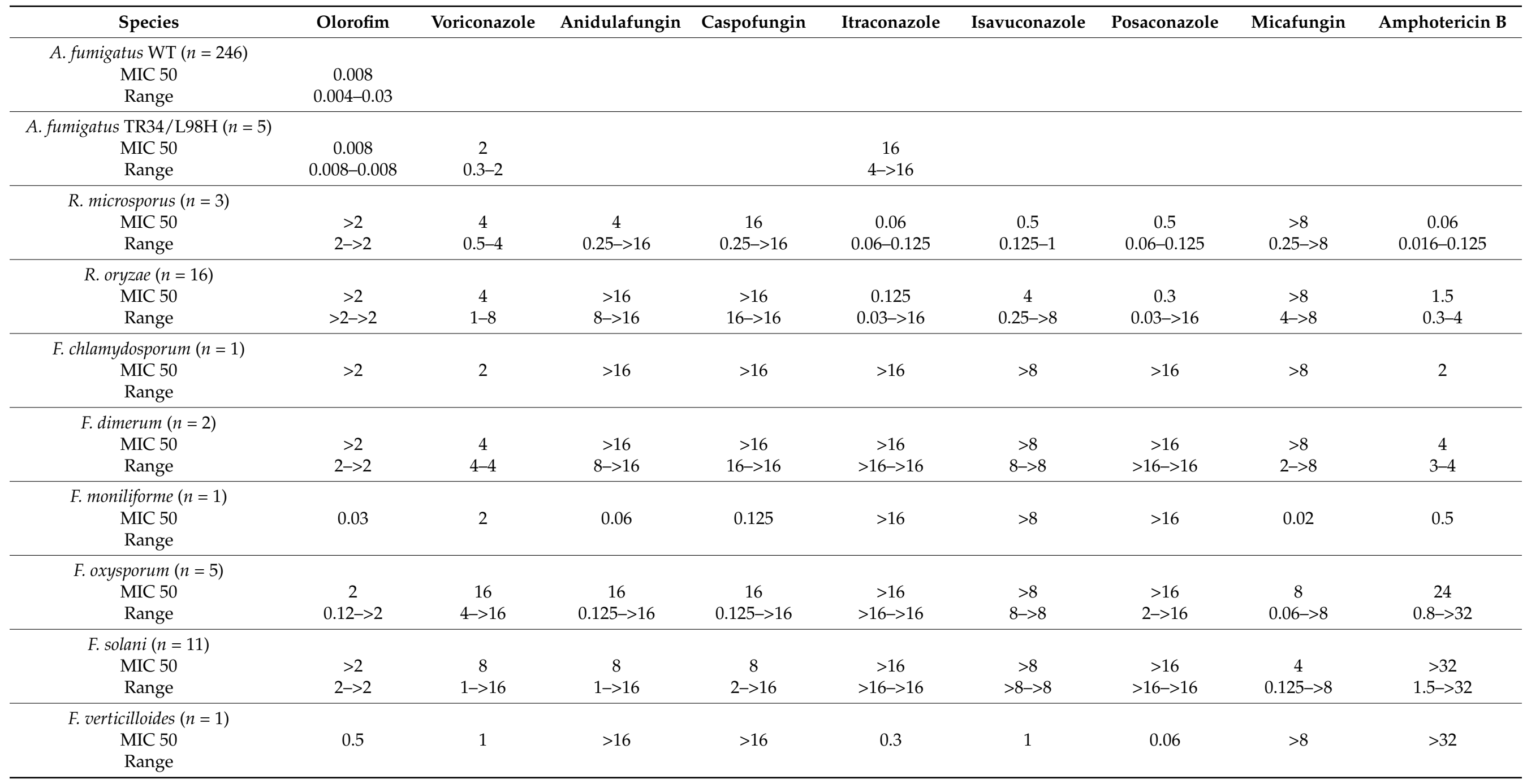


Table 2. Cont

\begin{tabular}{|c|c|c|c|c|c|c|c|c|c|}
\hline Species & Olorofim & Voriconazole & Anidulafungin & Caspofungin & Itraconazole & Isavuconazole & Posaconazole & Micafungin & Amphotericin B \\
\hline $\begin{array}{l}\text { M. anisopliae }(n=1) \\
\text { MIC } 50 \\
\text { Range }\end{array}$ & 0.5 & 1 & 0.125 & 0.06 & $>16$ & 8 & $>16$ & $<0.008$ & $>32$ \\
\hline $\begin{array}{l}\text { P. parasiticum }(n=1) \\
\text { MIC } 50 \\
\text { Range }\end{array}$ & 2 & 1 & 8 & $>16$ & $>16$ & 8 & 0.5 & $>8$ & 0.3 \\
\hline $\begin{array}{l}\text { P. curvatum }(n=1) \\
\text { MIC } 50 \\
\text { Range }\end{array}$ & 2 & 1 & 8 & $>16$ & 0.5 & 8 & 0.125 & $>8$ & 0.125 \\
\hline $\begin{array}{l}\text { P. richardsiae }(n=1) \\
\text { MIC } 50 \\
\text { Range }\end{array}$ & 0.06 & & & & & & & & 0.4 \\
\hline $\begin{array}{c}\text { Ramularia species }(n=1) \\
\text { MIC } 50 \\
\text { Range }\end{array}$ & 0.015 & 1 & $<0.008$ & $<0.008$ & 0.5 & 1 & 0.3 & $<0.008$ & 16 \\
\hline $\begin{array}{c}\text { S. kiliense }(n=1) \\
\text { MIC } 50 \\
\text { Range }\end{array}$ & 0.5 & 2 & 8 & 0.5 & $>16$ & $>8$ & $>16$ & 4 & 12 \\
\hline
\end{tabular}




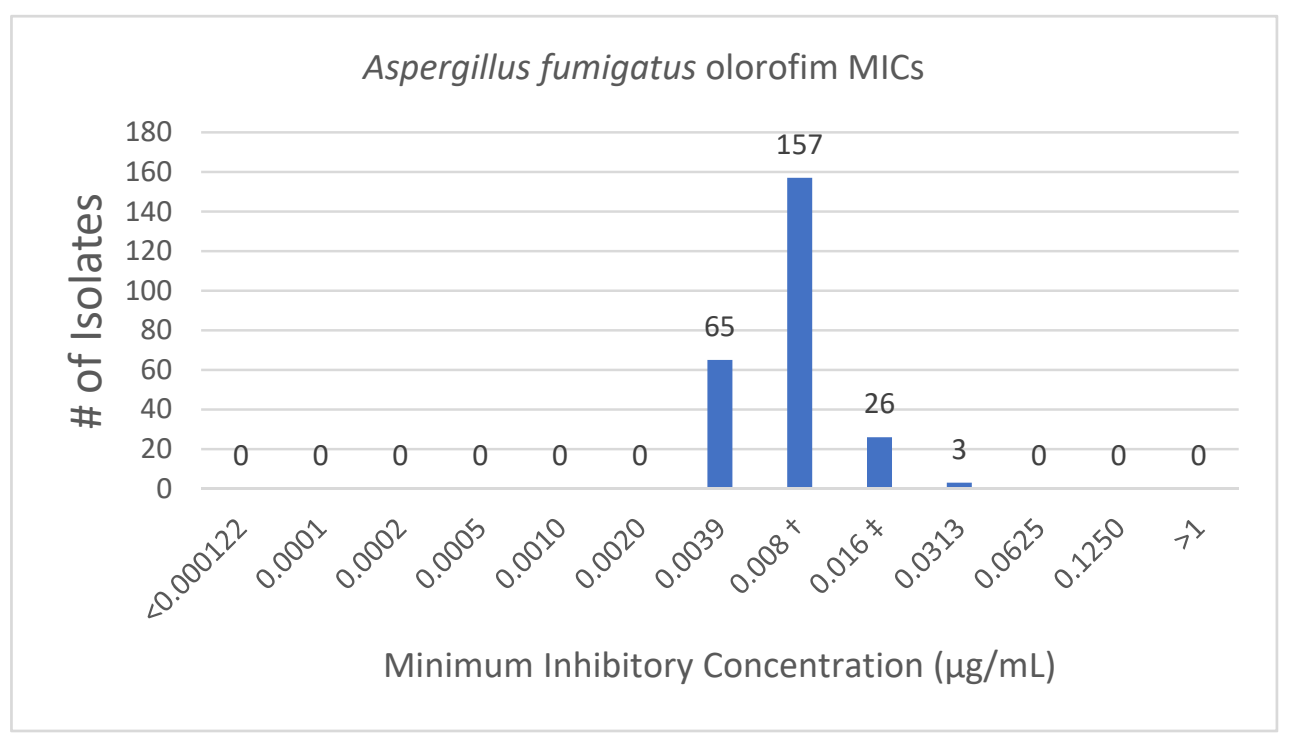

Figure 1. Distribution of olorofim MICs for Aspergillus fumigatus isolates. † MIC 50; $\ddagger$ MIC 90.

Olorofim showed variable antifungal activity amongst Fusarium spp. isolates. Olorofim showed no antifungal activity against $F$. chlamydosporum in the range tested (MIC $>2 \mu \mathrm{g} / \mathrm{mL}$ ). F. dimerum and F. solani species complex MICs were in the upper range of tested concentrations or showed no antifungal effect (MIC range 2 to $>2 \mu \mathrm{g} / \mathrm{mL}$ ). Olorofim had a wide range of antifungal activity against F. oxysporum (MIC $=0.12$ to $>2 \mu \mathrm{g} / \mathrm{mL}$ ). F. moniliforme and F. verticilloides had low olorofim MICs compared to other Fusarium spp. tested (MIC $=0.03$ and $0.50 \mu \mathrm{g} / \mathrm{mL}$, respectively). Phialemonium curvatum and Phaeoacremonium parasiticum were in the upper range tested (MIC $=2 \mu \mathrm{g} / \mathrm{mL}$ ), while Sarocladium kiliense (MIC $=0.5 \mu \mathrm{g} / \mathrm{mL}$ ), Ramularia spp. (MIC $=0.015 \mu \mathrm{g} / \mathrm{mL}$ ), Metarrhizium anisopliae $(\mathrm{MIC}=0.5 \mu \mathrm{g} / \mathrm{mL})$, and Pleurostomophora richardsiae $(\mathrm{MIC}=0.06 \mu \mathrm{g} / \mathrm{mL})$ all had low olorofim MIC values. Olorofim did not show in vitro inhibitory activity against Rhizopus microsporus or Rhizopus oryzae in the range tested, in agreement with other studies that show olorofim is ineffective against members of the Mucorales group.

\subsubsection{Azoles}

The collection was tested against the following azoles: isavuconazole, itraconazole, posaconazole, and voriconazole. Aside from $A$. fumigatus, voriconazole showed little antifungal activity against most isolates in this collection although the MICs to $R$. microsporus and A. fumigatus with mutations in $\mathrm{TR}_{34} / \mathrm{L} 98 \mathrm{H}$ and $\mathrm{TR}_{34} / \mathrm{L} 98 \mathrm{H} / \mathrm{S} 297 \mathrm{~T} / \mathrm{F} 495 \mathrm{I}$ were variable. For itraconazole, isolates of $R$. oryzae had variable MICs (range $0.03 \mu \mathrm{g} / \mathrm{mL}$ to $>16 \mu \mathrm{g} / \mathrm{mL}$ ) while all other species had only high MICs. R. microsporus, F. verticillioides, and Ramularia species all had low MICs to isavuconazole $(\leq 1 \mu \mathrm{g} / \mathrm{mL})$, however isavuconazole was not as effective against the remaining species, all of which had MIC in the upper range ( $\geq 8 \mu \mathrm{g} / \mathrm{mL}$ ). Consistent with the results for itraconazole, $R$. oryzae had variable MICs to isavuconazole (range $0.25 \mu \mathrm{g} / \mathrm{mL}$ to $>8 \mu \mathrm{g} / \mathrm{mL}$ ). For posaconazole, $R$. microsporus, F. verticilloides, P. curvatum, P. parasiticum, and Ramularia species all had low MICs $(\leq 0.5 \mu \mathrm{g} / \mathrm{mL})$. All other species had high MICs to posaconazole ( $\geq 2 \mu \mathrm{g} / \mathrm{mL}$ ) except for $R$. oryzae, which had variable MICs (range $0.03 \mu \mathrm{g} / \mathrm{mL}$ to $>16 \mu \mathrm{g} / \mathrm{mL}$ ).

\subsubsection{Echinocandins}

The collection was tested against anidulafungin, caspofungin, and micafungin. Minimal effective concentrations (MECs) were high for all three echinocandins, $\geq 2 \mu \mathrm{g} / \mathrm{mL}$ for R. oryzae, F. chlamydosporum, F. dimerum, F. verticilloides, P. curvatum, and P. parasiticum. For S. kiliense, R. microsporus, F. oxysporum, and Fusarium solani species complex echinocandin 
MECs were variable (range $0.06 \mu \mathrm{g} / \mathrm{mL}$ to $>16 \mu \mathrm{g} / \mathrm{mL}$ ). The remainder of the collection displayed low MECs to the echinocandins $(\leq 0.125 \mu \mathrm{g} / \mathrm{mL})$.

\subsubsection{Amphotericin B}

Amphotericin B was more effective than either the azoles or echinocandins. Rhizopus microsporus, F. moniliforme, P. curvatum, P. parasiticum, and P. richardsiae all exhibited low MICs to amphotericin B $(\leq 0.5 \mu \mathrm{g} / \mathrm{mL})$. Fusarium chlamydosporum, F. dimerum, F. verticilloides, S. kiliense, Ramularia species, and Metarrhizium anisopliae all exhibited high MICs to amphotericin B ( $\geq 2 \mu \mathrm{g} / \mathrm{mL}$ ). The MIC values for R. oryzae, F. oxysporum, and F. solani species complex were variable (range $0.25 \mu \mathrm{g} / \mathrm{mL}$ to $>32 \mu \mathrm{g} / \mathrm{mL}$ ).

\section{Discussion}

Our study corroborates previous findings showing the in vitro efficacy of olorofim against $A$. fumigatus and provides new data on rare and unusual molds. Previous studies exploring efficacy of olorofim against Aspergillus spp. found similar MIC ranges and susceptibility patterns. In Jørgensen et al., 235 A. fumigatus isolates were tested using the EUCAST method, resulting in a geometric mean MIC of $0.037 \mu \mathrm{g} / \mathrm{mL}$, which is consistent with our results despite the differences in testing methodology [20,34]. Buil et al. tested 10 A. fumigatus WT isolates and reported an olorofim $\mathrm{MIC}_{50}$ of $0.06 \mu \mathrm{g} / \mathrm{mL}$, the olorofim MICs of isolates with the $\mathrm{TR}_{34} / \mathrm{L} 98 \mathrm{H}$ mutation were in the same range as the WT isolates $(0.031-0.125 \mathrm{mg} / \mathrm{L})$ [18]. As olorofim has a completely different target to the azoles, crossresistance would not be expected. Consistent with this, an in-silico model predicts olorofim to possess a low probability of developing resistance in A. fumigatus [35]. A limitation of this study is the small sample size $(n=5)$ of $A$. fumigatus isolates with the $\mathrm{TR}_{34} / \mathrm{L} 98 \mathrm{H}$ mutation that were tested. The addition of our large collection of contemporary A. fumigatus isolates contributes to our knowledge of the wild type MIC range of this species against olorofim and will help with the establishment of epidemiological cutoff values. In addition, it shows that US isolates of A. fumigatus have similar susceptibility to olorofim as European isolates.

Jørgensen et al. used the EUCAST method to test olorofim susceptibility against different species of Fusarium and found F. dimerum and F. solani to have MICs $>1 \mu \mathrm{g} / \mathrm{mL}$ [20]. Our study tested multiple species of Fusarium and found elevated MICs of $\geq 2 \mu \mathrm{g} / \mathrm{mL}$ for all species except F. verticillioides (MIC $0.50 \mu \mathrm{g} / \mathrm{mL}$ ) and an isolate of $F$. oxysporum. Wiederhold et al. similarly found $F$. verticillioides had low MIC values with a range of $0.03-0.125 \mu \mathrm{g} / \mathrm{mL}$, indicating olorofim is likely to be active against $F$. verticillioides, but in many cases Fusarium are not identified to species so this observation may not be clinically relevant [36]. It was also shown that olorofim activity against Fusarium is endpoint dependent, and that using a 50\% inhibition endpoint results in lower MICs [36].

Rhizopus species remain difficult to treat and can have very high MIC values to amphotericin B and posaconazole [17,37-39]. Isavuconazole is the only antifungal with a US Food and Drug Administration indication for use against Mucorales. A recent study looked at $R$. microsporus susceptibility to olorofim and found no antifungal activity ( $n=4$, MIC $\geq 1$ ) [20]. The lack of antifungal activity of olorofim against Mucorales species can be explained by differences in the DHODH drug target. Mucorales' DHODH is distantly related to the DHODH of susceptible fungal species [19]. Dematiaceous and other rare molds can cause serious infections and are difficult to treat as many antifungals have little activity and effective treatment information is lacking. We included several rare molds from clinical cases of fungal infection to highlight the possible range of olorofim activity beyond the most commonly seen fungal infections. The activity of olorofim cannot be generalized for these rare infections but has shown good activity for some species and warrants further investigation. In conclusion we have confirmed the efficacy of olorofim against both WT and azole-resistant $A$. fumigatus seen in other studies as well demonstrated in vitro efficacy of olorofim against several Fusarium spp. and rare molds. 
Author Contributions: Conceptualization, E.L.B., O.G., N.S.N., E.M.R., M.B. and D.L.; Methodology, E.L.B., O.G., N.S.N. and E.M.R.; Software, N.S.N.; Validation, N.S.N.; Formal Analysis, O.G. and S.R.L.; Investigation, O.G.; Data Curation, E.M.R.; Writing-Original Draft Preparation, O.G. and N.S.N.; Writing-Review \& Editing, O.G., S.R.L., E.L.B., E.M.R., M.B. and D.L.; Supervision, S.R.L. and E.L.B.; Project Administration, E.L.B. and S.R.L. All authors have read and agreed to the published version of the manuscript.

Funding: This research received no external funding.

Institutional Review Board Statement: The isolates used in this study were approved for use by the Institutional Review Board of the Centers for Disease Control and Prevention.

Informed Consent Statement: Not applicable.

Data Availability Statement: Not applicable.

Acknowledgments: We are grateful to the members of the Mycotic Diseases Branch (MDB) at the Centers for Disease Control and Prevention (CDC) for their support.

Conflicts of Interest: M.B. and D.L. are employees and shareholders of F2G Ltd.

Disclaimer: The findings and the conclusions in this report are those of the authors and do not necessarily represent the view of the Centers for Disease Control and Prevention. Use of trade names if for identification only and does not imply endorsement.

\section{References}

1. Pfaller, M.A.; Pappas, P.G.; Wingard, J.R. Invasive Fungal Pathogens: Current Epidemiological Trends. Clin. Infect. Dis. 2006, 43, S3-S14. [CrossRef]

2. Rauseo, A.M.; Coler-Reilly, A.; Larson, L.; Spec, A. Hope on the Horizon: Novel Fungal Treatments in Development. Open Forum Infect Dis. 2020, 7, ofaa016. [CrossRef] [PubMed]

3. Vallabhaneni, S.; Mody, R.K.; Walker, T.; Chiller, T. The Global Burden of Fungal Diseases. Infect Dis. Clin. N. Am. 2016, 30 , 1-11. [CrossRef]

4. Brown, G.D.; Denning, D.W.; Gow, N.A.; Levitz, S.M.; Netea, M.G.; White, T.C. Hidden killers: Human fungal infections. Sci. Transl. Med. 2012, 4, 165rv13. [CrossRef]

5. Kosmidis, C.; Denning, D.W. The clinical spectrum of pulmonary aspergillosis. Thorax 2015, 70, 270-277. [CrossRef] [PubMed]

6. Verweij, P.E.; Mellado, E.; Melchers, W.J. Multiple-triazole-resistant aspergillosis. N. Engl. J. Med. 2007, 356, 1481-1483. [CrossRef]

7. Lestrade, P.P.A.; Meis, J.F.; Melchers, W.J.G.; Verweij, P.E. Triazole resistance in Aspergillus fumigatus: Recent insights and challenges for patient management. Clin. Microbiol. Infect. 2019, 25, 799-806. [CrossRef] [PubMed]

8. Enoch, D.A.; Yang, H.; Aliyu, S.H.; Micallef, C. The Changing Epidemiology of Invasive Fungal Infections. Methods Mol. Biol. 2017, 1508, 17-65. [PubMed]

9. Safdar, A.; Ma, J.; Saliba, F.; Dupont, B.; Wingard, J.R.; Hachem, R.Y.; Mattiuzzi, G.N.; Chandrasekar, P.H.; Kontoyiannis, D.P.; Rolston, K.V.; et al. Drug-induced nephrotoxicity caused by amphotericin B lipid complex and liposomal amphotericin B: A review and meta-analysis. Medicine (Baltimore) 2010, 89, 236-244. [CrossRef]

10. Townsend, R.; Dietz, A.; Hale, C.; Akhtar, S.; Kowalski, D.; Lademacher, C.; Lasseter, K.; Pearlman, H.; Rammelsberg, D.; Schmitt-Hoffman, A.; et al. Pharmacokinetic Evaluation of CYP3A4-Mediated Drug-Drug Interactions of Isavuconazole with Rifampin, Ketoconazole, Midazolam, and Ethinyl Estradiol/Norethindrone in Healthy Adults. Clin. Pharmacol. Drug. Dev. 2017, 6, 44-53. [CrossRef]

11. Verweij, P.E.; Chowdhary, A.; Melchers, W.J.; Meis, J.F. Azole Resistance in Aspergillus fumigatus: Can We Retain the Clinical Use of Mold-Active Antifungal Azoles? Clin. Infect. Dis. 2016, 62, 362-368. [CrossRef] [PubMed]

12. Perlin, D.S.; Rautemaa-Richardson, R.; Alastruey-Izquierdo, A. The global problem of antifungal resistance: Prevalence, mechanisms, and management. Lancet Infect. Dis. 2017, 17, e383-e392. [CrossRef]

13. Chowdhary, A.; Kathuria, S.; Xu, J.; Meis, J.F. Emergence of azole-resistant aspergillus fumigatus strains due to agricultural azole use creates an increasing threat to human health. PLoS Pathog. 2013, 9, e1003633. [CrossRef]

14. Resendiz Sharpe, A.; Lagrou, K.; Meis, J.F.; Chowdhary, A.; Lockhart, S.R.; Verweij, P.E. Triazole resistance surveillance in Aspergillus fumigatus. Med. Mycol. 2018, 56 (Suppl. S1), 83-92. [CrossRef]

15. Hurst, S.F.; Berkow, E.L.; Stevenson, K.L.; Litvintseva, A.P.; Lockhart, S.R. Isolation of azole-resistant Aspergillus fumigatus from the environment in the south-eastern USA. J. Antimicrob. Chemother. 2017, 72, 2443-2446. [CrossRef]

16. Wiederhold, N.P. Antifungal resistance: Current trends and future strategies to combat. Infect. Drug Resist. 2017, 10, 249-259. [CrossRef] [PubMed]

17. Dannaoui, E. Antifungal resistance in mucorales. Int. J. Antimicrob. Agents 2017, 50, 617-621. [CrossRef] [PubMed]

18. Buil, J.B.; Rijs, A.J.M.M.; Meis, J.F.; Birch, M.; Law, D.; Melchers, W.J.G.; Verweij, P.E. In vitro activity of the novel antifungal compound F901318 against difficult-to-treat Aspergillus isolates. J. Antimicrob. Chemother. 2017, 72, 2548-2552. [CrossRef] [PubMed] 
19. Oliver, J.D.; Sibley, G.E.M.; Beckmann, N.; Dobb, K.S.; Slater, M.J.; McEntee, L.; du Pré, S.; Livermore, J.; Bromley, M.J.; Wiederhold, N.P.; et al. F901318 represents a novel class of antifungal drug that inhibits dihydroorotate dehydrogenase. Proc. Natl. Acad. Sci. USA 2016, 113, 12809-12814. [CrossRef]

20. Jørgensen, K.M.; Astvad, K.M.T.; Hare, R.K.; Arendrup, M.C. EUCAST Determination of Olorofim (F901318) Susceptibility of Mold Species, Method Validation, and MICs. Antimicrob. Agents Chemother. 2018, 62, e00487-18. [CrossRef]

21. Wiederhold, N.P.; Najvar, L.K.; Jaramillo, R.; Olivo, M.; Birch, M.; Law, D.; Rex, J.H.; Catano, G.; Patterson, T.F. The Orotomide Olorofim Is Efficacious in an Experimental Model of Central Nervous System Coccidioidomycosis. Antimicrob. Agents Chemother. 2018, 62, e00999-18. [CrossRef]

22. du Pré, S.; Beckmann, N.; Almeida, M.C.; Sibley, G.E.M.; Law, D.; Brand, A.C.; Birch, M.; Read, N.D.; Oliver, J.D. Effect of the Novel Antifungal Drug F901318 (Olorofim) on Growth and Viability of Aspergillus fumigatus. Antimicrob. Agents Chemother. 2018, 62, e00231-18. [CrossRef] [PubMed]

23. Rivero-Menendez, O.; Cuenca-Estrella, M.; Alastruey-Izquierdo, A. In vitro activity of olorofim (F901318) against clinical isolates of cryptic species of Aspergillus by EUCAST and CLSI methodologies. J. Antimicrob. Chemother. 2019, 74, 1586-1590. [CrossRef]

24. Seyedmousavi, S.; Chang, Y.C.; Law, D.; Birch, M.; Rex, J.H.; Kwon-Chung, K.J. Efficacy of Olorofim (F901318) against Aspergillus fumigatus, A. nidulans, and A. tanneri in Murine Models of Profound Neutropenia and Chronic Granulomatous Disease. Antimicrob. Agents Chemother. 2019, 63, e00129-19. [CrossRef] [PubMed]

25. Lim, W.; Eadie, K.; Konings, M.; Rijnders, B.; Fahal, A.H.; Oliver, J.D.; Birch, M.; Verbon, A.; van de Sande, W. Madurella mycetomatis, the main causative agent of eumycetoma, is highly susceptible to olorofim. J. Antimicrob. Chemother. 2020, 75, 936-941. [CrossRef] [PubMed]

26. Biswas, C.; Law, D.; Birch, M.; Halliday, C.; Sorrell, T.C.; Rex, J.; Slavin, M.; Chen, S.C. In vitro activity of the novel antifungal compound F901318 against Australian Scedosporium and Lomentospora fungi. Med. Mycol. 2018, 56, 1050-1054. [CrossRef]

27. Talbot, J.J.; Frisvad, J.C.; Meis, J.F.; Hagen, F.; Verweij, P.E.; Hibbs, D.E.; Lai, F.; Groundwater, P.W.; Samson, R.A.; Kidd, S.E.; et al. cyp51A Mutations, Extrolite Profiles, and Antifungal Susceptibility in Clinical and Environmental Isolates of the Aspergillus viridinutans Species Complex. Antimicrob. Agents Chemother. 2019, 63, e00632-19. [CrossRef] [PubMed]

28. Lackner, M.; Birch, M.; Naschberger, V.; Grässle, D.; Beckmann, N.; Warn, P.; Gould, J.; Law, D.; Lass-Flörl, C.; Binder, U. Dihydroorotate dehydrogenase inhibitor olorofim exhibits promising activity against all clinically relevant species within Aspergillus section Terrei. J. Antimicrob. Chemother. 2018, 73, 3068-3073. [CrossRef]

29. Kennedy, T.; Graham, A.; Steiner, J. An open-label study in healthy volunteers to evaluate the potential for cytochrome P450 3A4 inhibition by F901318 using oral midazolam as a probe. In Proceedings of the ECCMID, Vienna, Austria, $22-25$ April 2017.

30. Guinea, J.; Verweij, P.E.; Meletiadis, J.; Mouton, J.W.; Barchiesi, F.; Arendrup, M.C. How to: EUCAST recommendations on the screening procedure E.Def 10.1 for the detection of azole resistance in Aspergillus fumigatus isolates using four-well azole-containing agar plates. Clin. Microbiol. Infect. 2019, 25, 681-687. [CrossRef] [PubMed]

31. Institute CaLS. Reference Method for Broth Dilution Antifungal Susceptibility Testing of Filamentous Fungi, M38, 3rd ed.; Clinical and Laboratory Standards Institute: Wayne, PA, USA, 2017.

32. Berkow, E.L.; Nunnally, N.S.; Bandea, A.; Kuykendall, R.; Beer, K.; Lockhart, S.R. Detection of TR 34 /L98H CYP51A Mutation through Passive Surveillance for Azole-Resistant Aspergillus fumigatus in the United States from 2015 to 2017. Antimicrob. Agents Chemother. 2018, 62, e02240-17. [CrossRef]

33. Smith, K.P.; Kirby, J.E. Verification of an Automated, Digital Dispensing Platform for At-Will Broth Microdilution-Based Antimicrobial Susceptibility Testing. J. Clin. Microbiol. 2016, 54, 2288-2293. [CrossRef] [PubMed]

34. Arendrup, M.C.; Meletiadis, J.; Mouton, J.W.; Guinea, J.; Cuenca-Estrella, M.; Lagrou, K.; Howard, S.J. Subcommittee on Antifungal Susceptibility Testing (AFST) of the ESCMID European Committee for Antimicrobial Susceptibility Testing (EUCAST). EUCAST technical note on isavuconazole breakpoints for Aspergillus, itraconazole breakpoints for Candida and updates for the antifungal susceptibility testing method documents. Clin. Microbiol. Infect. 2016, 22, 571. [PubMed]

35. Buil, J.B.; Oliver, J.; Law, D.; Tehupeiory-Kooreman, M.; Rex, J.H.; Hokken, M.W.J.; Melchers, W.J.G.; Birch, M.; Verweij, P.E. Molecular Mechanism and frequency of olorofim resistance in Aspergillus fumigatus. In Proceedings of the Trends in Medical Mycology bi-annual meeting of the European Confederation of Medical Mycology (ECMM), Nice, France, 11-14 October 2019.

36. Wiederhold, N.P.; Patterson, H.P.; Birch, M.; Law, D.; Rex, J. Evaluation of the In Vitro Activity of Olorofim against Fusarium Species. In Proceedings of the Trends in Medical Mycology bi-annual meeting of the European Confederation of Medical Mycology (ECMM), Nice, France, 11-14 October 2019.

37. Vehreschild, J.J.; Birtel, A.; Vehreschild, M.J.; Liss, B.; Farowski, F.; Kochanek, M.; Sieniawski, M.; Steinbach, A.; Wahlers, K.; Fätkenheuer, G.; et al. Mucormycosis treated with posaconazole: Review of 96 case reports. Crit. Rev. Microbiol. 2013, 39, 310-324. [CrossRef]

38. Marty, F.M.; Ostrosky-Zeichner, L.; Cornely, O.A.; Mullane, K.M.; Perfect, J.R.; Thompson, G.R., 3rd; Alangaden, G.J.; Brown, J.M.; Fredricks, D.N.; Heinz, W.J.; et al. VITAL and FungiScope Mucormycosis Investigators. Isavuconazole treatment for mucormycosis: A single-arm open-label trial and case-control analysis. Lancet Infect. Dis. 2016, 16, 828-837. [CrossRef]

39. Skiada, A.; Lass-Floerl, C.; Klimko, N.; Ibrahim, A.; Roilides, E.; Petrikkos, G. Challenges in the diagnosis and treatment of mucormycosis. Med. Mycol. 2018, 56 (Suppl. S1), 93-101. [CrossRef] [PubMed] 